\title{
Fatal dysfunction and disintegration of thrombin-stimulated platelets
}

Haematologica 2019

Volume 104(9):1866-1878

\section{Correspondence: \\ JOHN WEISEL \\ weisel@pennmedicine.upenn.edu \\ Received: July 18, 2018. \\ Accepted: February 14, 2019. \\ Pre-published: February 21, 2019.}

doi:10.3324/haematol.2018.202309

Check the online version for the most updated information on this article, online supplements, and information on authorship \& disclosures: www.haematologica.org/content/104/9/1866

(C)2019 Ferrata Storti Foundation

Material published in Haematologica is covered by copyright. All rights are reserved to the Ferrata Storti Foundation. Use of published material is allowed under the following terms and conditions:

https://creativecommons.org/licenses/by-nc/4.0/legalcode. Copies of published material are allowed for personal or internal use. Sharing published material for non-commercial purposes is subject to the following conditions:

https://creativecommons.org/licenses/by-nc/4.0/legalcode, sect. 3. Reproducing and sharing published material for commercial purposes is not allowed without permission in writing from the publisher.
Oleg V. Kim, ${ }^{1,2}$ Tatiana A. Nevzorova, ${ }^{3}$ Elmira R. Mordakhanova, ${ }^{3}$ Anastasia A. Ponomareva, ${ }^{3,4}$ Izabella A. Andrianova, ${ }^{3}$ Giang Le Minh, ${ }^{3}$ Amina G. Daminova, ${ }^{3,4}$ Alina D. Peshkova, ${ }^{3}$ Mark S. Alber, ${ }^{2}$ Olga Vagin,,$^{5,6}$ Rustem I. Litvinov $^{1,3}$ and John W. Weisel ${ }^{1}$

${ }^{1}$ University of Pennsylvania Perelman School of Medicine, Department of Cell and Developmental Biology, Philadelphia, PA, USA; '2University of California Riverside, Department of Mathematics, Riverside, CA, USA; ${ }^{3}$ Kazan Federal University, Institute of Fundamental Medicine and Biology, Kazan, Russian Federation; ${ }^{4}$ Kazan Institute of Biochemistry and Biophysics, FRC Kazan Scientific Center of RAS, Kazan, Russian Federation; ${ }^{5}$ Geffen School of Medicine at UCLA, Department of Physiology, Los Angeles, CA, USA and ${ }^{6}$ VA Greater Los Angeles Healthcare System, Los Angeles, CA, USA

\section{ABSTRACT}

$\mathrm{P}$ latelets play a key role in the formation of hemostatic clots and obstructive thrombi as well as in other biological processes. In response to physiological stimulants, including thrombin, platelets change shape, express adhesive molecules, aggregate, and secrete bioactive substances, but their subsequent fate is largely unknown. Here we examined late-stage structural, metabolic, and functional consequences of thrombin-induced platelet activation. Using a combination of confocal microscopy, scanning and transmission electron microscopy, flow cytometry, biochemical and biomechanical measurements, we showed that thrombin-induced activation is followed by time-dependent platelet dysfunction and disintegration. After $\sim 30$ minutes of incubation with thrombin, unlike with collagen or $\mathrm{ADP}$, human platelets disintegrated into cellular fragments containing organelles, such as mitochondria, glycogen granules, and vacuoles. This platelet fragmentation was preceded by $\mathrm{Ca}^{2+}$ influx, integrin $\alpha_{\mathrm{II}} \beta_{3}$ activation and phosphatidylserine exposure (activation phase), followed by mitochondrial depolarization, generation of reactive oxygen species, metabolic ATP depletion and impairment of platelet contractility along with dramatic cytoskeletal rearrangements, concomitant with platelet disintegration (death phase). Coincidentally with the platelet fragmentation, thrombin caused calpain activation but not activation of caspases 3 and 7. Our findings indicate that the late functional and structural damage of thrombin-activated platelets comprise a calpain-dependent platelet death pathway that shares some similarities with the programmed death of nucleated cells, but is unique to platelets, therefore representing a special form of cellular destruction. Fragmentation of activated platelets suggests that there is an underappreciated pathway of enhanced elimination of platelets from the circulation in (pro)thrombotic conditions once these cells have performed their functions.

\section{Introduction}

Platelets are blood cells that play a pivotal role in preventing bleeding (hemostasis) and obstructing blood vessels (thrombosis), in addition to other biological functions. ${ }^{1,2}$ Activated platelets mechanically contract blood clots and thrombi, ${ }^{3}$ which is an important pathogenic mechanism in thrombosis. ${ }^{46}$ Under (patho)physiological conditions, platelets activated by stimulants change their morphology, express adhesive molecules, undergo aggregation, and secrete bioactive substances. Disruption of these functions can have pathological consequences, including heart attack and stroke. However, the mechanisms underlying the subsequent fate of activated platelets, including platelet clearance, remain poorly defined. The structural and func- 
tional consequences of platelet activation and the survival of these cells are related to fundamental aspects of cell biology, including death pathways of anucleate cells.

One of the main platelet activators is thrombin, which causes exposure of activated adhesive proteins (integrins $\alpha_{\mathrm{II}} \beta_{3}, \alpha_{v} \beta_{3}, \alpha_{2} \beta_{1}$, P-selectin, ephrins, etc.), secretion, contraction, and changes in energy metabolism..$^{711}$ Stimulation leads to the platelets changing from a discoid shape to a star-like cell with multiple protrusions, with this morphological change being driven by reorganization of actin, tubulin, spectrin, and filamin. ${ }^{12-16}$ Another major consequence of platelet activation is the release of microvesicles segregated into plasma membrane-derived ectosomes and exosomes, originating from intracellular structures, both of which have important biological functions. ${ }^{17,18}$

Apoptosis plays an essential role in the survival of platelets in vivo. ${ }^{19}$ The role of apoptosis in platelet lifespan was discovered as a result of the use of BH3-mimetic anticancer drugs, which induce apoptosis in cancer cells, but also cause thrombocytopenia. ${ }^{20}$ The role of apoptosis in platelet lifespan has been delineated mainly through various mouse models. ${ }^{19}$ In contrast, the fate of platelets after activation is still mired in controversy ${ }^{21}$ and, therefore, deserves further study.

In this study we tested the hypothesis that thrombininduced platelet activation later results in metabolic exhaustion, dysfunction, and breakup of platelets. Using light and electron microscopy combined with flow cytometry and rheometry, we revealed dynamic alterations of platelet morphology and cytoskeletal rearrangements that accompany biochemical and biomechanical changes in platelets treated with thrombin. Thrombin causes delayed agonistspecific dose-dependent platelet dysfunction and fragmentation associated with reorganization of actin. Thrombininduced exposure of phosphatidylserine and active integrin $\alpha_{\mathrm{II}} \beta_{3}$, as well as $\mathrm{Ca}^{2+}$ influx characteristic of initial platelet activation, are followed by mitochondrial depolarization, formation of reactive oxygen species and metabolic ATP depletion concomitant with platelet disintegration and activation of calpain, but not effector caspases, suggesting a calpain-dependent pathway of platelet death.

\section{Methods}

Blood was collected and processed in accordance with a protocol approved by the University of Pennsylvania Institutional Review Board and in compliance with the Helsinki Declaration of ethical principles for medical research involving human subjects.

Formation of platelet-rich plasma (PRP) clots, distinct fluorescent labeling of components of the PRP clots for confocal microscopy, dynamic rheometry of contracting clots, platelet isolation, staining of actin and other intracellular structures, scanning and transmission electron microscopy, flow cytometry, measurement of ATP and mitochondrial transmembrane potential, caspase and calpain activity, western blot, image analysis and statistical analyses are described in the Online Supplementary Methods.

\section{Results}

\section{Thrombin-induced fragmentation of platelets revealed with confocal microscopy and flow cytometry}

Confocal microscopy of platelets in fresh, hydrated plasma clots formed with thrombin and $\mathrm{Ca}^{2+}$ revealed that, fol- lowing shape changes characteristic of platelet activation, many platelets and small platelet aggregates fell apart into subcellular particles in a time-dependent manner. One hour after addition of thrombin, a substantial fraction ( $25 \%)$ of platelets underwent disintegration, revealed as various stages of fragmentation (Figure 1A,B). After $3 \mathrm{~h}$ of thrombin-induced activation, multiple platelet fragments $(>90 \%$ of platelets) were dispersed within the fibrin network (Figure 1C). Notably, the platelet fragments displayed a bimodal size distribution with two distinct peaks at about $200 \mathrm{~nm}$ and $900 \mathrm{~nm}$ (Figure 1D), which are much smaller than intact platelets $(2-4 \mu \mathrm{m})$. The platelet fragmentation observed by microscopy started $\sim 30$ min following thrombin treatment and progressed linearly (Figure 1E). The degree of platelet fragmentation was dose-dependent in the range of 0.1-10 U/mL thrombin (Online Supplementary Figure $S 1 A$ ) and was partially inhibited by rivaroxaban, an inhibitor of factor Xa (Online Supplementary Figure S1B), suggesting the combined destructive effect on platelets of added and endogenously generated thrombin. Remarkably, the onset of platelet fragmentation was the same at $1 \mathrm{U} / \mathrm{mL}$ and $10 \mathrm{U} / \mathrm{mL}$ concentrations of thrombin, suggesting that platelet disintegration is a delayed process that occurs only after activation is completed and platelets enter a new (dys)functional stage. Flow cytometry of thrombin-treated platelets revealed that the number of CD41-positive fragments smaller than $1 \mu \mathrm{m}$ increased 10- to 20 -fold after 60 min (Figure 1F). Concomitantly, the number of platelets (gated as CD41-positive particles $>1 \mu \mathrm{m}$ ) decreased 2.5- to 3 -fold compared to the number of untreated platelets (Figure 1G).

Time-lapse confocal microscopy of platelets in PRP clots revealed that after initial activation, individual platelets and their aggregates attached to fibrin and extended filopodia to pull on fibrin fibers, causing clot contraction that lasted about $30 \mathrm{~min}$. Subsequently, platelets began decomposing into subcellular fragments (Figure 2). There were two types of platelet-derived particles, which differed in size and cellular origin. One type separated from the ends of the filopodia, while the other type arose from the cell bodies undergoing fragmentation. The tips of filopodia formed smaller platelet fragments (Figure 2A), while much larger subcellular fragments parted from the platelet bodies (Figure 2A,B).

\section{Ultrastructural visualization of thrombin-induced platelet fragmentation}

To see structural details of thrombin-induced platelet fragmentation, isolated platelets treated with thrombin for 15 and $60 \mathrm{~min}$ and control untreated platelets were imaged using transmission and scanning electron microcopy (Figure 3). Resting platelets displayed a typical discoid shape with a smooth plasma membrane and cytoplasm containing mitochondria, glycogen granules, lysosomes, an open canalicular system, and dense and $\alpha$-granules (Figure 3A,C). In contrast, many platelets treated with thrombin for 15 min broke apart into more or less separated membranous particles, some of which contained inclusions, such as mitochondria, glycogen granules and vacuoles (Figure 3B, see fragments 1-4). Scanning electron microscopy of thrombintreated platelets revealed multiple blebs on the surface, likely reflecting fragmentation of the cell body (Figure 3E), quite differently from the untreated control (Figure 3G). At the longer incubation time with thrombin (60 min), platelets underwent further disintegration, producing small fragments (Figure 3D,F) with size distributions peaking at 430 
$\mathrm{nm}$ according to both the transmission and scanning electron micrographs (Figure 3H,I). Importantly, platelets treated with soluble collagen or ADP maintained their integrity during $2 \mathrm{~h}$ of incubation, although they did show morphological signs of activation (Online Supplementary Figure S2).

\section{Cytoskeletal rearrangements associated with} thrombin-induced platelet fragmentation

To see whether fragmentation of thrombin-activated platelets involves rearrangement of actin cytoskeleton, we used fluorescent confocal microscopy to follow the dynamics of rhodamine-labeled F-actin in resting platelets and thrombin-treated platelets for $1 \mathrm{~h}$. In most resting platelets, F-actin was detected throughout the area of a cell section and was distributed evenly rather than forming patches (Figure 4A and Online Supplementary Figure $S 3 A$ ). In some resting platelets, F-actin was preferentially distributed along the cell periphery. By contrast, in thrombin-activated platelets F-actin was typically detected as intense fluorescent clusters shifted toward the cell center
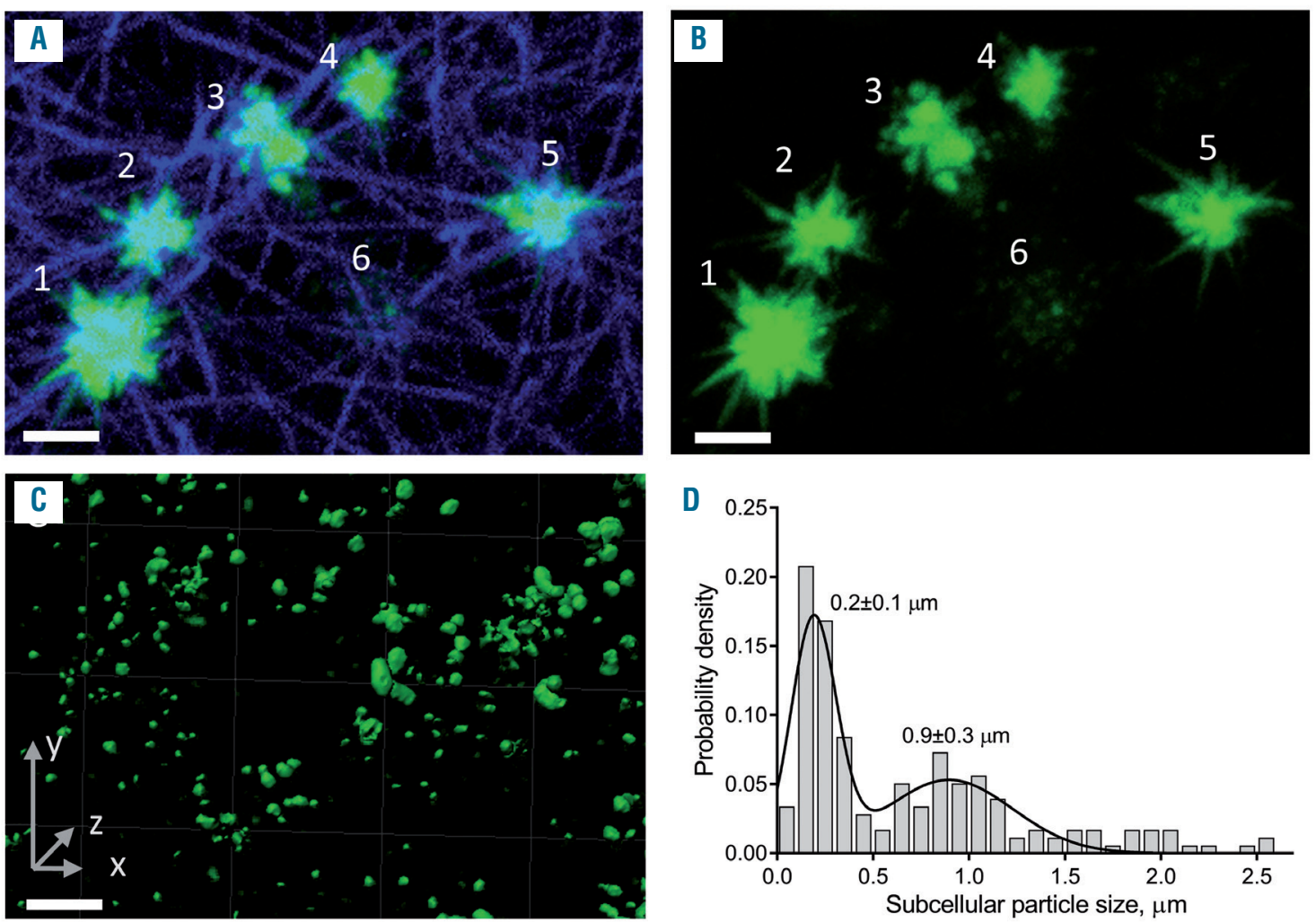

E

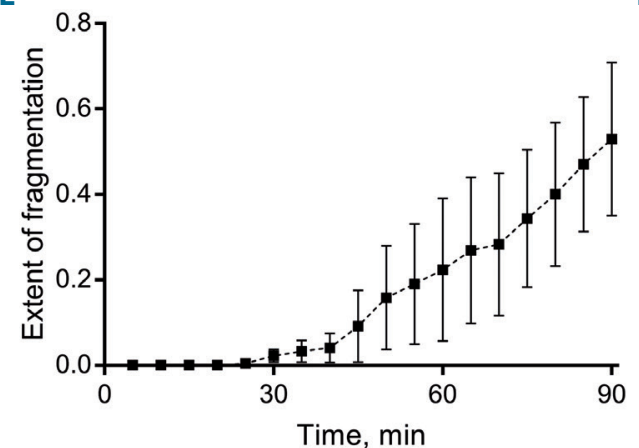

$\mathrm{F}$

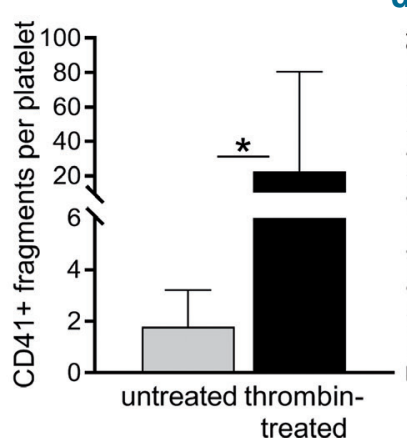

G

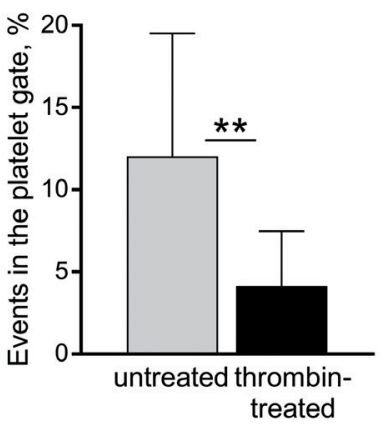

Figure 1. Thrombin-induced platelet fragmentation. (A-C) Representative confocal microscopy images of thrombin-activated platelets undergoing shape changes and fragmentation. The images were acquired $1 \mathrm{~h}(\mathrm{~A}, \mathrm{~B})$ and $3 \mathrm{~h}(\mathrm{C})$ after clotting of citrated human platelet-rich plasma (PRP) with $1 \mathrm{U} / \mathrm{mL}$ thrombin and $31 \mathrm{mM} \mathrm{CaCl}$ at $37^{\circ} \mathrm{C}$. (A) Fibrin-attached activated platelets with filopodia $(1,2,5)$ and platelets at different stages of fragmentation: initial (4), intermediate (3), and final (6). Platelets are labeled with calcein (green) and fibrin is labeled with Alexa Fluor-647 (blue). (B) The same platelets as in (A) are shown without the fibrin channel. (see Online Supplementary Movie S1 for the thrombin-induced fragmentation dynamics at a large scale in three dimensions). (C) Confocal microscopy-based three-dimensional reconstruction of widespread fragmentation of thrombin-treated platelets within a PRP clot prepared as in (A) after $3 \mathrm{~h}$ of incubation. Scale bars in $(\mathrm{A}-\mathrm{C})=3$ um. (See Online Supplementary Movie S2 for the large-scale three-dimensional reconstructed image). (D) The subcellular particle size distribution in a PRP clot ( $n=180$, bars) fitted with a multi-peak Gaussian function (solid line). (E) Increasing fraction of fragmented platelets in a PRP clot under conditions described in (A-C) determined from time-lapse confocal microscopy (mean \pm standard error of mean, $n=4$ ). (F) Flow cytometry-based enumeration of platelet-derived CD41-positive platelet fragments normalized by the number of gated platelets after 60 min of incubation of isolated platelets with $1 \mathrm{U} / \mathrm{mL}$ thrombin in Tyrode buffer with $2 \mathrm{mM}$ $\mathrm{CaCl}_{2}$ at $37^{\circ} \mathrm{C}$ (mean \pm standard deviation, $\mathrm{n}=10$ ). (G) Relative numbers of the platelet-gated signals (corresponding to the intact platelets remaining in a sample) after 60 min of incubation under the same conditions as in $(\mathrm{E})$ (mean \pm standard deviation, $\mathrm{n}=12$ ). $* P<0.05, * * P<0.01$. 
(Figure 4B,D and Online Supplementary Figure S3B). F-actin was detected in platelet fragments but the fluorescence intensity was non-uniform (Figure 4E and Online Supplementary Figure S3C).

Inhibition of actin polymerization by latrunculin $\mathrm{A}$ or cytochalasin $\mathrm{D}$ before activation with thrombin prevented platelet fragmentation, as revealed by both confocal microscopy of platelets in plasma clots (Figure 4G,H) and transmission electron microscopy of isolated platelets (Online Supplementary Figure S4A-E). Remarkably, latrunculin $\mathrm{A}$ in combination with thrombin caused significant enlargement of the open canalicular system, associated with formation of large vacuoles as well as platelet degranulation, but without disintegration of platelets (Figure 4H and Online Supplementary Figure S4C,D). Pretreatment of platelets with paclitaxel, which inhibits depolymerization of microtubules, also prevented throm- bin-induced platelet disintegration (not shown), indicating involvement of the plasma membrane cytoskeleton built of microtubules.

\section{Condensation of F-actin in thrombin-stimulated} platelets and its extinction during fragmentation

In order to quantify changes in the intracellular concentration or compactness of actin, the intensity of the F-actin stain was compared in untreated and thrombin-treated cells, using confocal microscopy at identical microscope settings. Thrombin caused an $\sim 300$-fold increase in the overall intracellular F-actin intensity (Figure 4C), which was accompanied by a reduction in the size of individual platelets. The X-Y cross-sectional area of resting platelets was between 3 and $12 \mu \mathrm{m}^{2}$ with a median of $7.5 \mu \mathrm{m}^{2}$, while in the vast majority of thrombin-treated platelets, the cross-sectional area was less than $5 \mu \mathrm{m}^{2}$ with a median
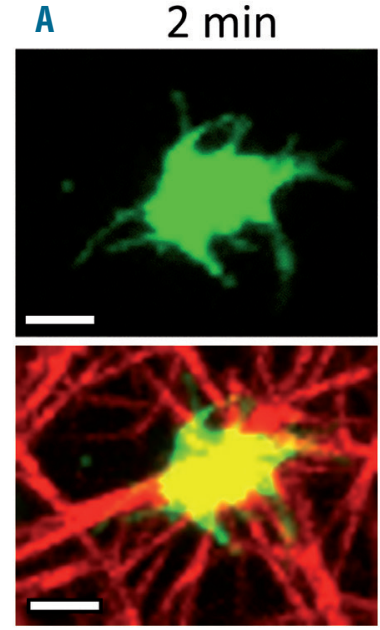

B

$2 \min$
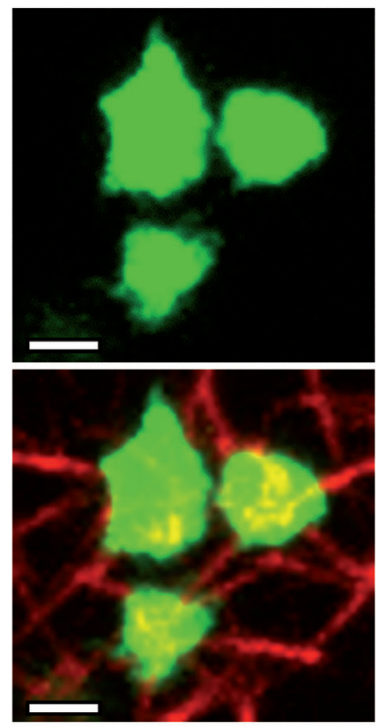

$6 \min$
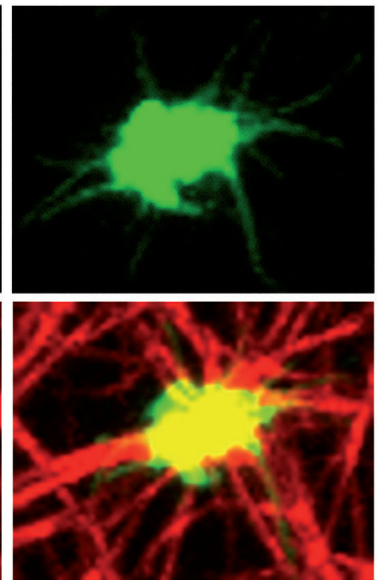

$16 \min$
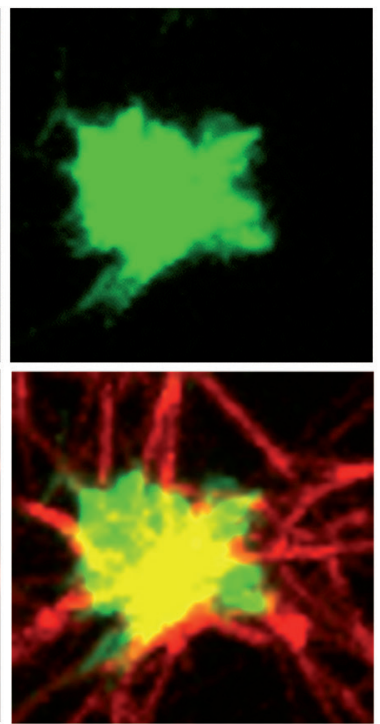

$20 \min$
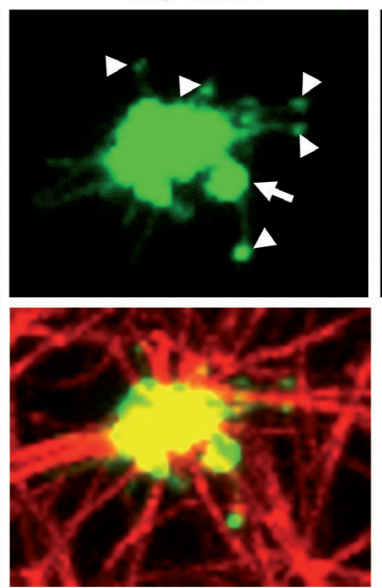

$24 \min$
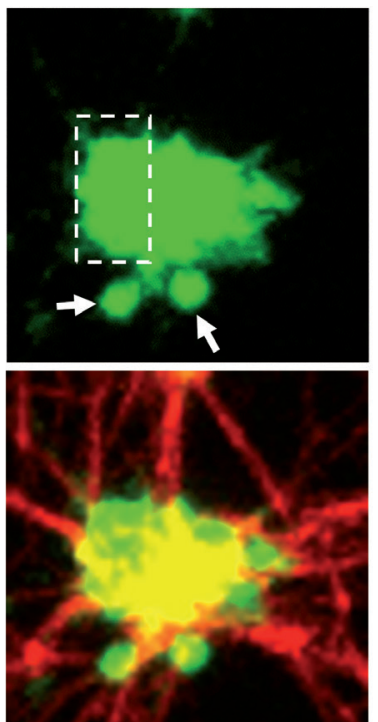

$33 \min$

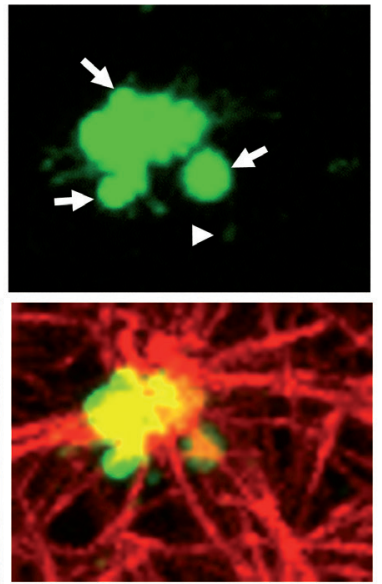

$60 \mathrm{~min}$
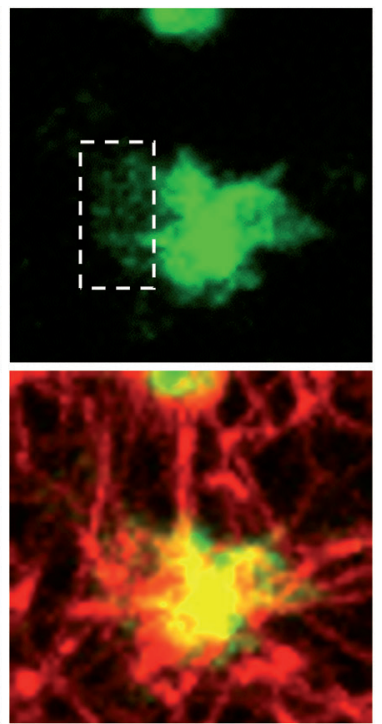

Figure 2. Time-lapse confocal microscopy of thrombin-induced platelet fragmentation in a plasma clot. (A) A single thrombin-activated platelet and (B) an aggregate of three thrombin-activated platelets fall apart and form cellular fragments over time. The upper rows in (A) and (B) show platelets without fibrin. Subcellular fragments originating from the filopodia (white arrowheads) and cell bodies (white arrows) are indicated. The dashed rectangular area in (B) at 24 min and 60 min timepoints show disintegration of a larger part of the platelet aggregate (24 min) into smaller fragments (60 min). Platelets are green and fibrin is red. Scale bars in (A) and $(B)=3 \mu \mathrm{m}$. 

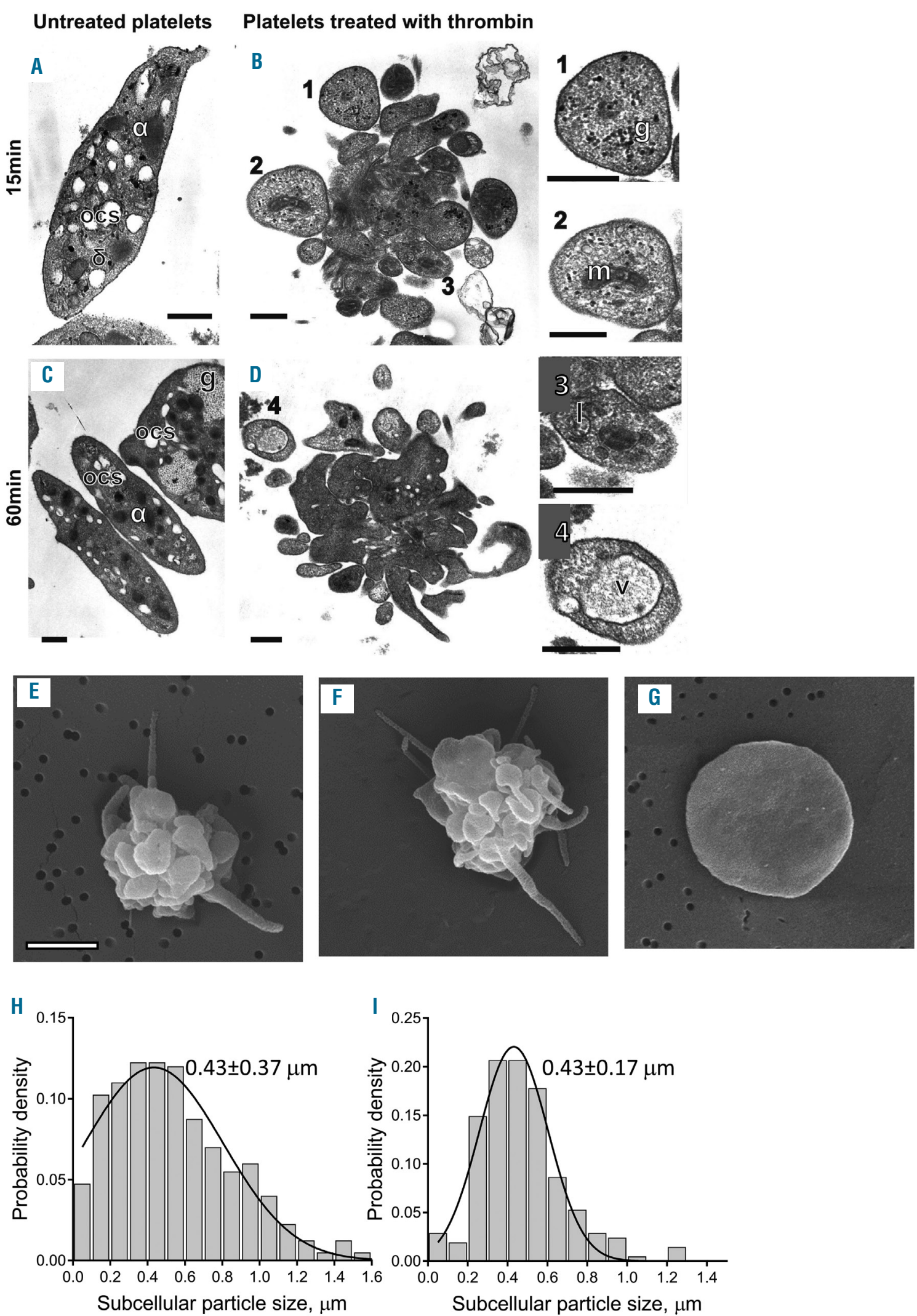

Figure 3. Ultrastructural characterization of thrombin-induced platelet fragments. (A-D) Representative transmission electron micrographs of isolated control untreated platelets (A, C) and platelets incubated with $1 \mathrm{U} / \mathrm{mL}$ of thrombin for $15 \mathrm{~min}$ (B) and $60 \mathrm{~min}$ (D) at $37^{\circ} \mathrm{C}$ in Tyrode buffer with $2 \mathrm{mM} \mathrm{CaCl}$. (1-4) Particles formed during thrombin-induced fragmentation of a platelet. The designated structures are $\alpha$-granules $(\alpha)$, $\delta$-granules $(\delta)$, glycogen granules (g), lysosomal vacuoles $(I)$, mitochondria $(\mathrm{m})$, open canalicular system (ocs), vacuole $(\mathrm{v})$. Scale bars in $(A-D)=0.5 \mu \mathrm{m}$. (E-G) Representative scanning electron micrographs of isolated platelets incubated at $37^{\circ} \mathrm{C}$ with $1 \mathrm{U} / \mathrm{mL}$ of thrombin for $15 \mathrm{~min}(\mathrm{E})$ and $60 \mathrm{~min}(\mathrm{~F})$ and a control untreated platelet $(\mathrm{G})$ in Tyrode buffer with $2 \mathrm{mM} \mathrm{CaCl}$. Scale bars in (E-G) $=1$ um. (H, I) Size distributions of thrombin-induced platelet fragments from micrographs obtained with transmission ( $\mathrm{H}, \mathrm{n}=400$ fragments) and scanning (I, $\mathrm{n}=210$ fragments) electron microscopy. The experimentally determined particle size distributions (bars) in (H) and (I) are fitted with a Gaussian function (solid lines). 

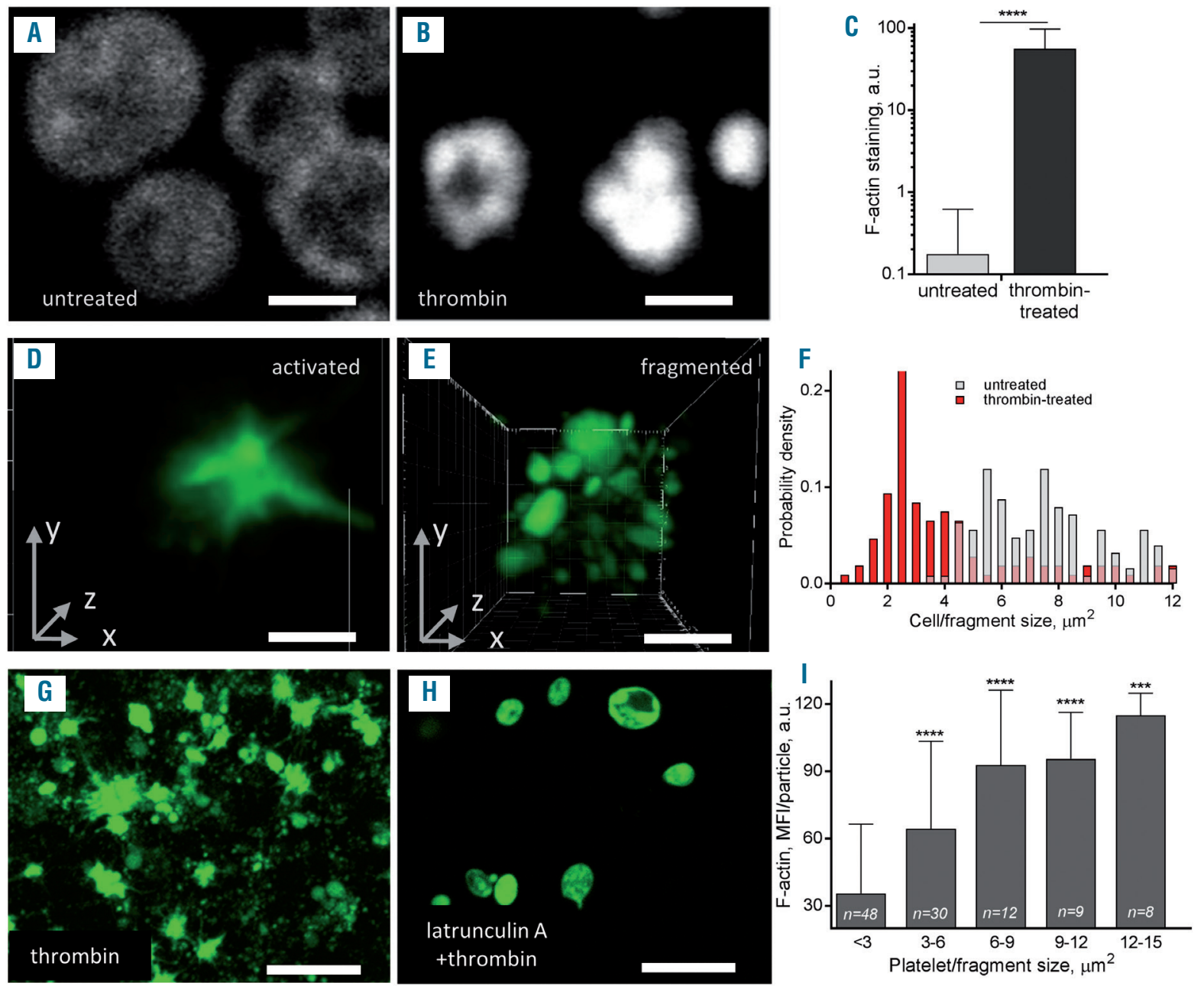

Figure 4. Rearrangement of actin during thrombin-induced platelet fragmentation. (A, B) Representative confocal microscopy z-stack projections of fixed isolated platelets stained for F-actin with Alexa Fluor 488-phalloidin before (A) and $60 \mathrm{~min}$ after (B) treatment with thrombin showing the intracellular redistribution of actin and a substantial increase in the intensity of the F-actin stain in the thrombin-treated platelets. Scale bars $=2 \mu \mathrm{m}$. (C) The F-actin-related fluorescence intensity in resting versus thrombin-treated platelets was quantified under identical staining conditions and microscope settings (mean \pm standard deviation, $n=3$ ). (D, E) F-actinbased three-dimensional reconstruction of thrombin-activated platelets in platelet-rich plasma (PRP) clots before (D) and after (E) fragmentation captured at 5 min and $60 \mathrm{~min}$ after addition of thrombin, respectively. Scale bars $=2 \mu \mathrm{m}$. (F) Histogram of F-actin-containing cellular particles of various size in untreated ( $\mathrm{n}=107$ ) and thrombin-treated ( $n=107$ ) platelets, showing a shift towards smaller platelet fragments after $1 \mathrm{~h}$ of incubation with thrombin. (G, H) Representative confocal microscopy z-stack projections of calcein-labeled platelets (green) treated with thrombin in the absence $(G)$ and presence $(H)$ of latrunculin $A$, which prevented platelet fragmentation. Scale bars $=10 \mu \mathrm{m}$. (I) Mean fluorescence intensity of individual whole platelets or cellular fragments stained for F-actin (total 107 particles analyzed) as a function of their size (mean \pm standard deviation). $* * * P<0.001$ and $* * * * P<0.0001$ compared to the untreated platelets, two-tailed Mann-Whitney $\mathrm{U}$ test.
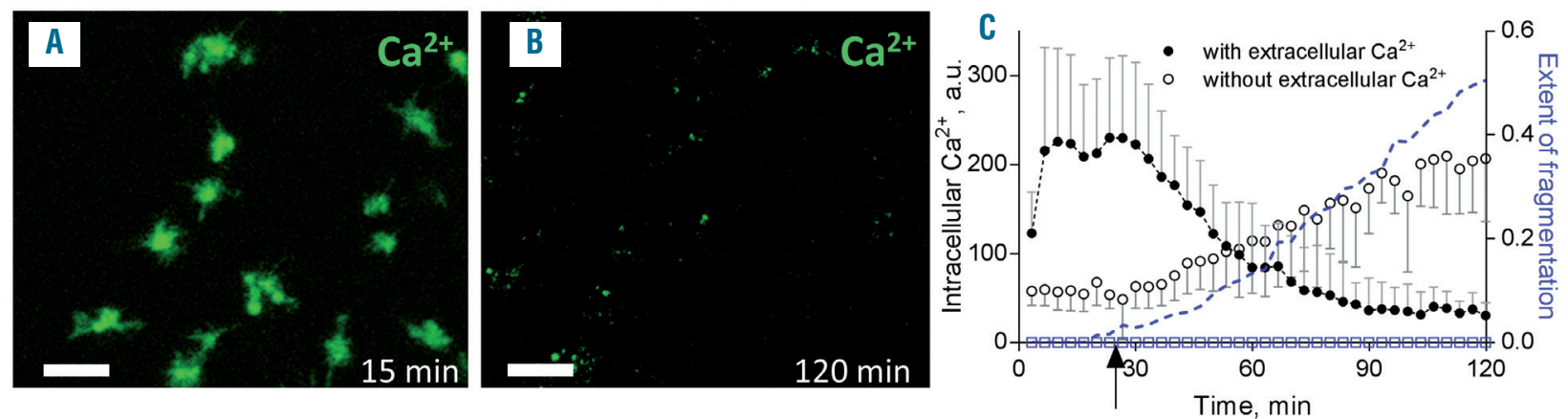

Figure 5. Time-lapse measurement of intracellular calcium in thrombin-treated platelets. (A, B) Representative confocal micrographs of platelets pre-incubated with a Ca ${ }^{2+}$-sensitive fluorophore Fluo-4AM at $15 \mathrm{~min}$ (A) and $120 \mathrm{~min}$ (B) after addition of thrombin to platelet-rich plasma in the presence of extracellular Ca ${ }^{2+}$. Scale bars $=4 \mu \mathrm{m}$. (C) Confocal microscopy-based dynamics of intracellular $\mathrm{Ca}^{2+}$ levels in thrombin-activated platelets (mean \pm standard deviation, $\mathrm{n}=3$ ) in the presence (black circles) and absence (open circles) of extracellular $\mathrm{Ca}^{2+}$ superimposed over the time course of platelet fragmentation in the presence (blue dashed line) and absence (blue open squares) of extracellular $\mathrm{Ca}^{2+}$. The arrow indicates the onset of platelet disintegration in the presence of $\mathrm{Ca}^{2+}$. There were statistically significant differences $(P<0.05)$ for $30 \mathrm{~min}$ versus $60 \mathrm{~min}$ and for $60 \mathrm{~min}$ versus $120 \mathrm{~min}$. 
of $3.5 \mu \mathrm{m}^{2}$ (Figure 4F). Remarkably, the intensity of F-actin staining decreased in fragments (Figure 4I), so that in many particles that had a cross-sectional area $<4 \mu \mathrm{m}^{2} \mathrm{~F}-$ actin staining was not detected at all, suggesting a breakdown of the actin network during platelet fragmentation. The high intensity of F-actin staining in thrombin-activated platelets and its extinction in fragments is clearly seen in three-dimensional reconstructions (Figure 4D,E).

\section{Thrombin-induced platelet fragmentation is preceded by $\mathrm{Ca}^{2+}$ influx and is $\mathrm{Ca}^{2+}$-dependent}

Because platelet functionality and structural rearrangements depend strongly on $\mathrm{Ca}^{2+}$, we correlated thrombininduced platelet fragmentation with intracellular $\mathrm{Ca}^{2+}$ concentration using confocal microscopy with a $\mathrm{Ca}^{2+}$-dependent fluorophore (Figure 5A,B). The intracellular $\mathrm{Ca}^{2+}$ spiked during the first minutes of thrombin treatment in the presence of extracellular calcium. After spiking, the average platelet calcium content remained constant up to about $30 \mathrm{~min}$, and then decreased greatly, with a strong, inverse correlation with the time course of platelet fragmentation $(r=-0.93, P<0.01)$. In contrast, in the absence of extracellular $\mathrm{Ca}^{2+}$, the intracellular calcium (released from intracellular depots) increased monotonically 4-fold after 120 min of incubation with thrombin, with no evidence of platelet fragmentation within $2 \mathrm{~h}$ (Figure 5C).

\section{Mitochondrial depolarization, ATP depletion, and generation of reactive oxygen species in platelets undergoing thrombin-induced fragmentation}

Thrombin induced a time-dependent reduction of the mitochondrial transmembrane potential $\left(\Delta \Psi_{\mathrm{m}}\right)$ in platelets. As revealed by time-lapse confocal microscopy, the overall fluorescence intensity of the $\Delta \Psi_{\mathrm{m}}$-sensitive MitoTracker dye in freshly formed thrombin-initiated PRP-clots dropped 2- and 4-fold after $60 \mathrm{~min}$ and $90 \mathrm{~min}$, respectively (Figure 6A-D). A similar gradual decrease was observed with another $\Delta \Psi_{\mathrm{m}}$-sensitive dye, tetramethylrhodamine (data not shown). Remarkably, in activated platelets some mitochondria were translocated towards the platelet periphery, localized within filopodia and either remained inside filopodia-derived vesicles or got released as free mitochondria into the extracellular space (Figure 6E,F). The reducing mitochondrial membrane potential strongly and inversely $(\mathrm{r}=-0.93, P<0.01)$ correlated with an increase of the fraction of disintegrated platelets, which reached $55 \%$ of the total number of visualized platelets by $90 \mathrm{~min}$ (Figure 6G). Remarkably, the initial drop of $\Delta \Psi_{\mathrm{m}}$ was almost concurrent with the beginning of platelet fragmentation at about 30 min after thrombin-induced clot formation and platelet activation. This time point also corresponded to the termination of contraction of a PRP clot, measured as a decrease by $90 \%$ of platelet-generated contractile stress (Figure $6 \mathrm{H}$ ), suggesting that platelet disintegration is responsible for the cessation of clot contraction.

To establish whether thrombin treatment disturbs energy metabolism, we performed time-lapse quantification of the ATP content in thrombin-treated platelets using confocal microscopy in the presence of an ATP-sensitive fluorophore. The intracellular ATP decreased progressively during the $2 \mathrm{~h}$ following platelet activation (Figure 6I). Importantly, such steady kinetics is characteristic of the metabolic depletion of ATP rather than ATP secretion, which occurs as a burst within the first seconds or minutes following platelet activation with thrombin. ${ }^{22}$
Accordingly, when ATP was measured both in platelet lysates and in activated platelet supernatants during a $3 \mathrm{~h}$ incubation of PRP with thrombin, the ATP in the supernatant first increased (over $15 \mathrm{~min}$ ), reflecting the fast ATP secretion from activated platelets, and then remained constant, while the intracellular ATP decreased progressively over time (Figure 6J). These results confirm that the continuing decrease in thrombin-treated platelet ATP is not due to secretion, but results from gradual metabolic exhaustion. The decline of the intracellular ATP level and reduction of $\Delta \Psi_{\mathrm{m}}$ in response to thrombin were concomitant with reactive oxygen species (ROS) formation, which occurred at about 30 min after activation (Figure 6K-M). Importantly, the increasing mitochondrial ROS production showed a strong temporal correlation ( $\mathrm{r}=-0.98$, $P<0.01$ ) with the platelet disintegration dynamics (Figure $6 \mathrm{M})$.

\section{Lack of caspase activation in platelets undergoing thrombin-induced fragmentation}

Previous studies suggested involvement of executor caspases in thrombin-induced platelet activation and further dysfunction. $^{23-25}$ To test whether thrombin-treated platelets underwent a caspase-dependent death pathway, we used time-lapse confocal microscopy of PRP clots and flow cytometry of thrombin-activated isolated platelets pre-incubated with a fluorogenic substrate of caspases 3 and 7 . In both experimental settings, there were no signs of caspase activation in response to thrombin stimulation, at least within $1.5 \mathrm{~h}$ (Figure 7A-F). Cleavage of the fluorogenic caspase substrate, as revealed by confocal microscopy, was detected in less than $3 \%$ of platelets after 90 min of treatment with thrombin (Figure 7A,C), while treatment for $90 \mathrm{~min}$ with calcium ionophore A23187, used as a positive control, led to the activation of caspases 3 and 7 in about one-third of platelets (Figure 7B,C). Flow cytometry also did not reveal any increase of caspase activity in response to thrombin treatment up to $3 \mathrm{~h}$, with less than $0.5 \%$ of platelets exhibiting substrate-related fluorescence, while stimulation of platelets with A23187 led to the activation of caspases in $32 \%$ of platelets (Figure $7 \mathrm{D}, \mathrm{E})$. It is worth noting that an increase of thrombin concentration from $1 \mathrm{U} / \mathrm{mL}$ to $5 \mathrm{U} / \mathrm{mL}$ did not cause caspase activation (data not shown). In addition, no procaspase 3 cleavage was revealed in western blots of platelet lysates obtained from thrombin-treated platelets (Figure 7G). The lack of caspase activation suggests that thrombin-induced platelet dysfunction and fragmentation represent a caspase-independent pathway of platelet death.

\section{Calpain activity increases in platelets undergoing thrombin-induced fragmentation}

As an alternative to caspases, calpains may also contribute to platelet fragmentation by cleaving cytoskeletal proteins such as actin and fodrin. ${ }^{26,27}$ We evaluated activity of calpains in thrombin-treated platelets incubated with a fluorogenic calpain substrate using both flow cytometry of isolated cells and time-lapse confocal microscopy of platelets in PRP clots. As revealed by flow cytometry, treatment of platelets with thrombin resulted in 1.5- and 2 -fold increases of a fluorescent signal of the calpain cleavage product after $60 \mathrm{~min}$ and $180 \mathrm{~min}$ incubation, respectively, as compared to that in untreated cells (Figure 8A). At the same time, the signal from the calpain cleavage product was 7-fold weaker than that of the strongly 

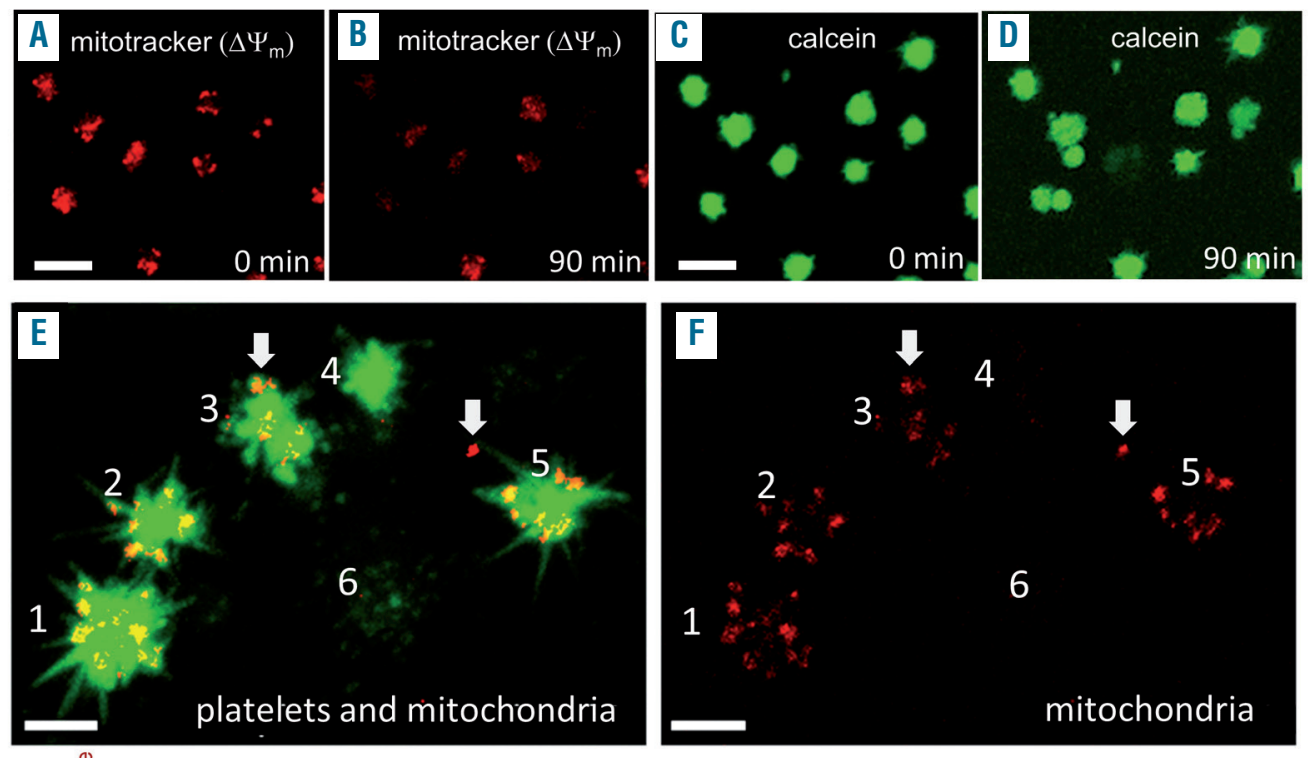

G
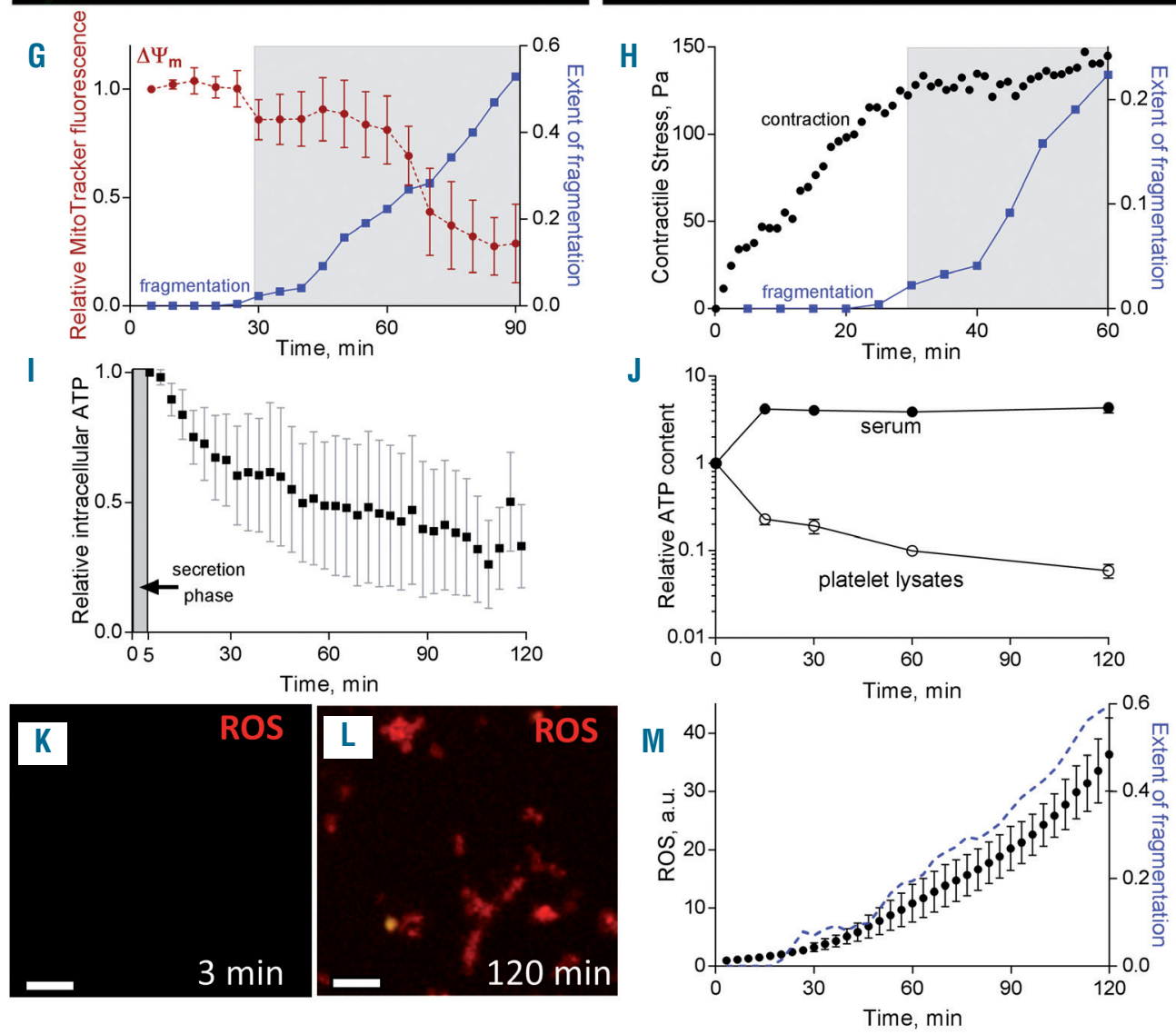

Figure 6. Concurrent thrombin-induced platelet fragmentation, cessation of contractility, mitochondrial depolarization, metabolic ATP depletion, and reactive oxygen species production. (A-D) Representative confocal microscopy images of platelet mitochondria stained with a red MitoTracker (A, B) and the same platelets marked with green calcein (C, D) before (A, C) and 90 min after (B, D) adding thrombin to platelet-rich plasma (PRP). (E, F) Confocal microscopy images showing both activated green platelets and red mitochondria $(E)$ or mitochondria only $(F)$ located at the platelet periphery and in filopodia $(1,2,5)$ as well as in separated platelet fragments $(3,4,6)$. White arrows indicate typical examples of a mitochondrion inside a platelet fragment and a free extracellular mitochondrion. Scale bars in $(A-D)=5 \mu m$, those in $(E)$ and $(\mathrm{F})=3 \mu \mathrm{m}$. (G) Time-lapse confocal microscopy of the $\Delta \Psi_{\mathrm{m}}$-sensitive MitoTracker fluorescence intensity (red symbols) (mean \pm standard error of mean, $\mathrm{n}=4$ ), reflecting a gradual decrease of $\Delta \Psi_{\mathrm{m}}$ inversely correlating with the increasing average fraction of fragmented platelets (blue symbols). There were statistically significant differences for the initial time point versus $30 \mathrm{~min}$ and for $30 \mathrm{~min}$ versus $90 \mathrm{~min}$ ( $P<0.05$, two-tailed Mann-Whitney $\mathrm{U}$ test). (H) Superimposition of the platelet-generated contractile stress over platelet fragmentation dynamics in contracting blood clots. The shaded areas in $(\mathrm{G})$ and $(\mathrm{H})$ show the time-frame of cessation of clot contraction concurrent with mitochondrial depolarization and fragmentation. (I) Gradual metabolic reduction of the intracellular ATP content in individual thrombin-stimulated platelets (mean \pm standard error of mean, 50-100 platelets analyzed from 3 PRP clots). There were statistically significant differences for the initial time point versus 30 min and for 30 min versus $120 \mathrm{~min}(\mathrm{P}<0.05$, two-tailed Mann-Whitney $\mathrm{U}$ test). $(\mathrm{J})$ Parallel dynamic measurements of the ATP content in lysates of thrombin-activated platelets and in the activated platelet supernatant following clot formation normalized by the thrombin-free baseline (mean \pm standard deviation, $\mathrm{n}=3$ ). There were statistically significant differences for the initial time point versus 30 min for supernatant, and for the initial time point versus 30 min and 30 min versus 120 min for platelet lysates $(P<0.05$, two tailed Mann-Whitney $\mathrm{U}$ test). Whole blood $(\mathrm{G}, \mathrm{H})$ or PRP $(\mathrm{I}, \mathrm{J})$ was activated with $1 \mathrm{U} / \mathrm{mL}$ thrombin and $\mathrm{CaCl}_{2}$ at $37^{\circ} \mathrm{C}$. (K, L) Representative confocal micrographs of platelets pre-incubated with MitoSOX ${ }^{\mathrm{TM}}$ Red (mitochondrial super oxide indicator) at $3 \mathrm{~min}(\mathrm{~K})$ and $60 \mathrm{~min}(\mathrm{~L})$ after addition of thrombin to PRP in the presence of Ca ${ }^{2+}$. Scale bars $=4 \mu \mathrm{m}$. (See Online Supplementary Movies S3 and S4 for the full $120 \mathrm{~min}$ long time sequences at low and high magnifications) (M) Confocal microscopy-based dynamics of reactive oxygen species (ROS) production superimposed on the fragmentation dynamics, both determined in PRP clots (mean \pm standard deviation, $n=3$ ). 
increased fluorescence observed after treatment of platelets with A23187, used as a positive control. In accordance with the flow cytometry data, the time-lapse fluorescent confocal microscopy of PRP clots pre-incubated for 90 min with a calpain substrate also revealed activation of platelet calpains in response to thrombin. Specifically, fluorescence of the calpain cleavage product detected in platelets 90 min after thrombin treatment was 6.5-fold higher than that detected in untreated platelets (Figure 8B).
Remarkably, the rate of enzymatic reaction calculated as the first derivative of the dynamic fluorescence signal reached a maximum (the highest calpain activity) after 35 min of thrombin-induced platelet activation, coinciding with a decrease in the mitochondrial membrane potential (Figure $8 \mathrm{C}$ ) and the beginning of platelet fragmentation (Figure 8D).

Accordingly, pre-treatment of platelets with a calpain inhibitor ALLN prior to platelet activation resulted in a 3-
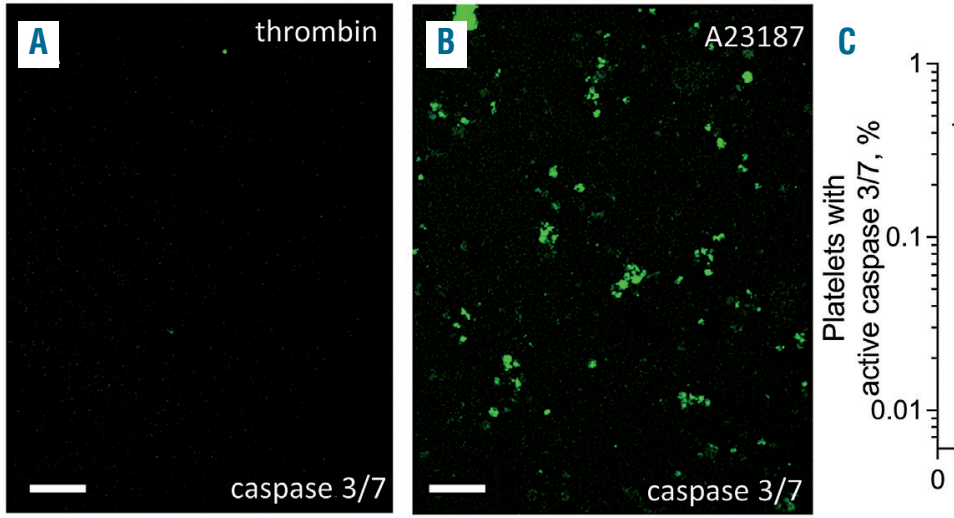

D
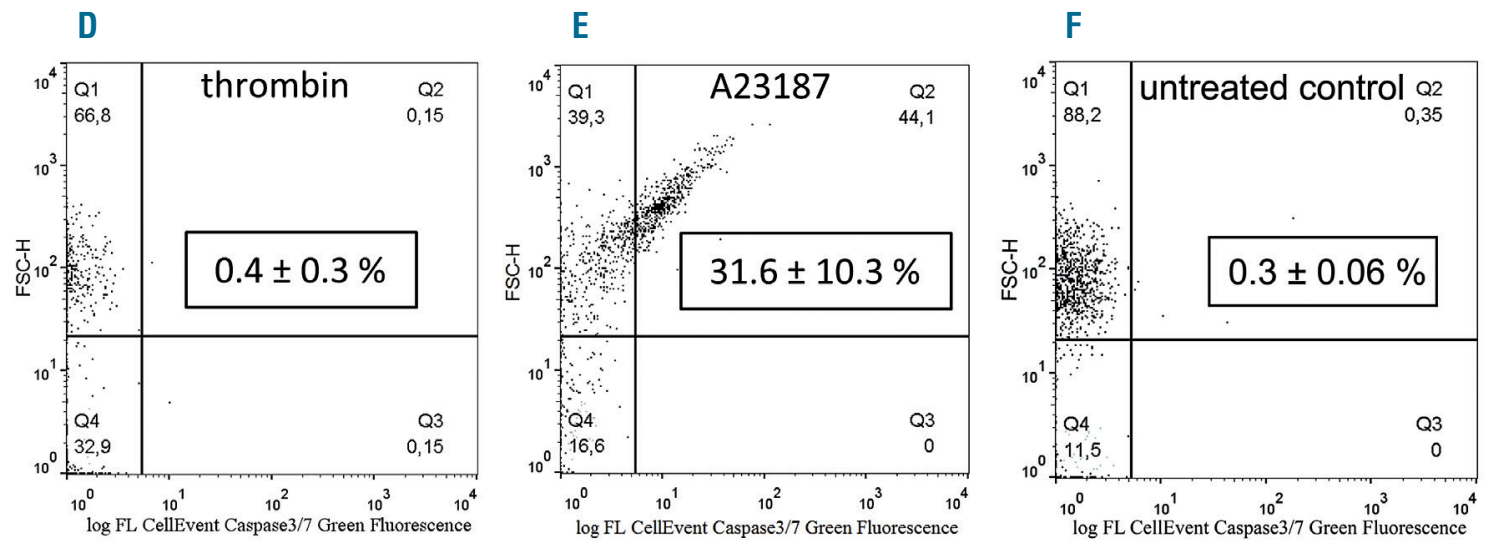

G

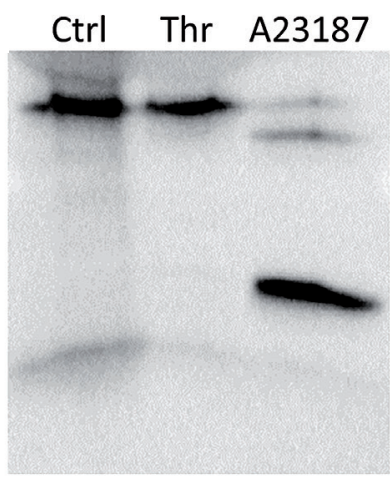

A23187 (positive control)

a- thrombin

o untreated control

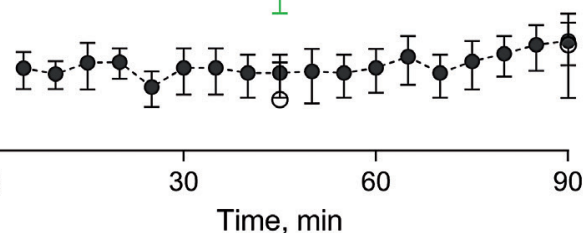

\section{Procaspase 3 \\ $(32 \mathrm{kDa})$}

Caspase 3

$(17 \mathrm{kDa})$

Figure 7. The lack of caspase 3/7 activity in thrombin-stimulated platelets. (A, B) Representative confocal micrographs of activated platelets pre-incubated with a fluorogenic substrate of caspases 3 and 7. Platelets were visualized after activation with thrombin (A) or the calcium ionophore A23187 (B, positive control) in platelet-rich plasma (PRP) clots in the presence of $\mathrm{Ca}^{2+}$. Scale bars $=4 \mu \mathrm{m}$. (C) Quantitative estimation of the dynamic caspase-3/7 activity in platelets based on confocal microscopy (mean \pm standard deviation, $n=3$ ). $P<0.01$ for the difference between thrombin-treated platelets and the positive control (two-tailed MannWhitney U test). (D-F) Representative flow cytometry dot plots of isolated platelets analyzed for caspase-3/7 activity with a fluorogenic substrate after incubation with thrombin (D), the calcium ionophore A23187 (E) and without treatment (F). The inset boxed numbers (mean \pm standard deviation) indicate the average caspase-3/7positive fractions of platelets stimulated with thrombin (D) and A23187 (E) and untreated platelets $(\mathrm{F})(\mathrm{n}=3, P<0.0001)$. (G) Western blot analysis of caspase-3 cleav age in platelet lysates obtained from control untreated platelets (Ctrl), platelets treated with thrombin (Thr) and platelets treated with the calcium ionophore A23187 used as a positive control. No caspase-3/7 activation was revealed in platelets treated with $1 \mathrm{U} / \mathrm{mL}$ thrombin at $37^{\circ} \mathrm{C}$ either in PRP (A-C) or Tyrode buffer (D-G) in the presence of $\mathrm{Ca}^{2+}$ 
fold decrease in the rate of accumulation of the calpain cleavage product (Figure 8B). The residual calpain activity in thrombin-treated platelets exposed to ALLN was still higher than that in untreated platelets because ALLN did not suppress calpain completely, while in the resting platelets calpain was not activated at all. Importantly, pretreatment of platelets with ALLN prior to platelet activation caused a significant initial increase in fluorescence of the $\Delta \Psi_{\mathrm{m}}$-sensitive MitoTracker by about $35 \%$ in freshly formed PRP clots (Figure 8C). Furthermore, the timedependent drop of $\Delta \Psi_{\mathrm{m}}$ was attenuated by ALLN; after 90 min of thrombin-induced platelet activation in the presence of ALLN, $\Delta \Psi_{\mathrm{m}}$ was reduced by only $20 \%$, while without the calpain inhibitor the reduction of $\Delta \Psi_{\mathrm{m}}$ was $65 \%(P<0.05)$.

To establish whether active calpain is involved in platelet fragmentation, platelets were observed over time in PRP clots in the absence or presence of ALLN (Figure 8D). Calpain inhibition caused an $~ 30$ min delay in the commencement of thrombin-induced platelet fragmentation, which was followed by an increase in the number of fragmented cells at about the same rate as in the absence of ALLN (27\%/min vs. $28 \% / \mathrm{min}$ with and without ALLN, respectively), suggesting that calpain is involved in platelet fragmentation during the first $30 \mathrm{~min}$, followed by seemingly calpain-independent platelet disintegration. After 90 min of incubation, the fraction of thrombin-activated fragmented platelets pre-treated with ALLN was $55 \%$ smaller than the fraction of disintegrated platelets in the absence of ALLN. Thus, inhibition of calpain partially protects thrombin-treated platelets from a progressive decrease of $\Delta \Psi_{\mathrm{m}}$ and delays fragmentation, suggesting involvement of calpain in thrombin-induced platelet dysfunction and disintegration.

\section{Discussion}

While the early stages of platelet activation have been studied extensively, ${ }^{2,710}$ alterations of platelets occurring at the later stages, after their functions have been fulfilled, are much less well understood. Meanwhile, such late-
A

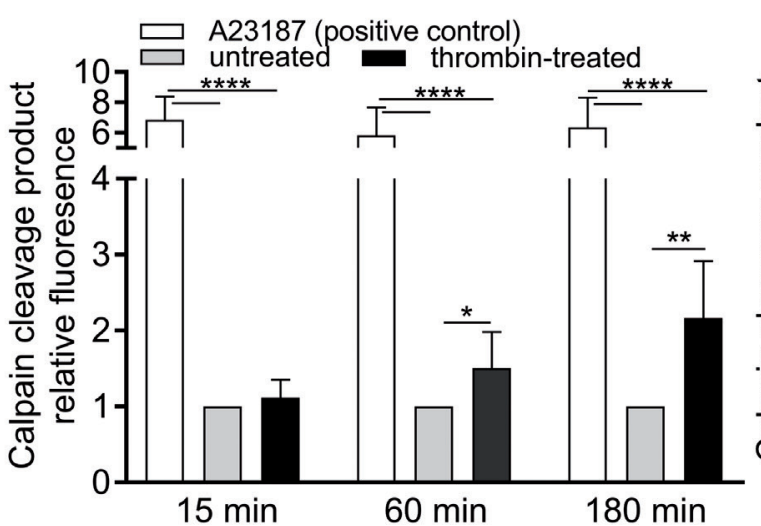

C

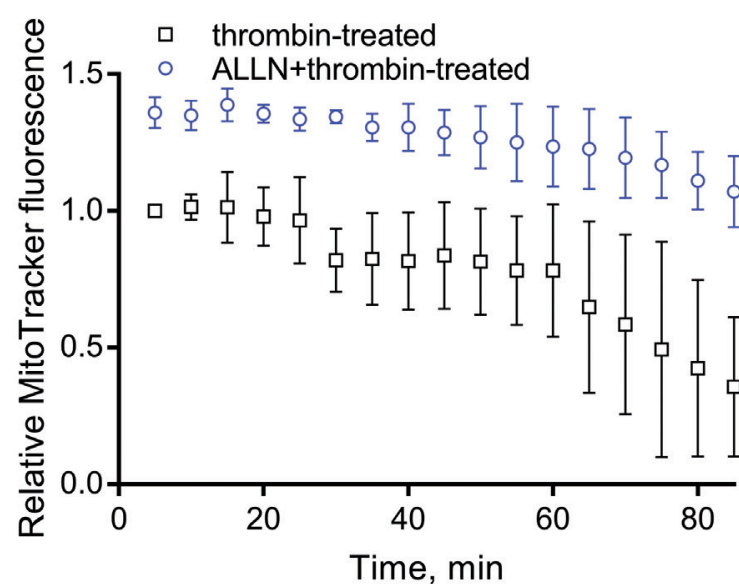

B
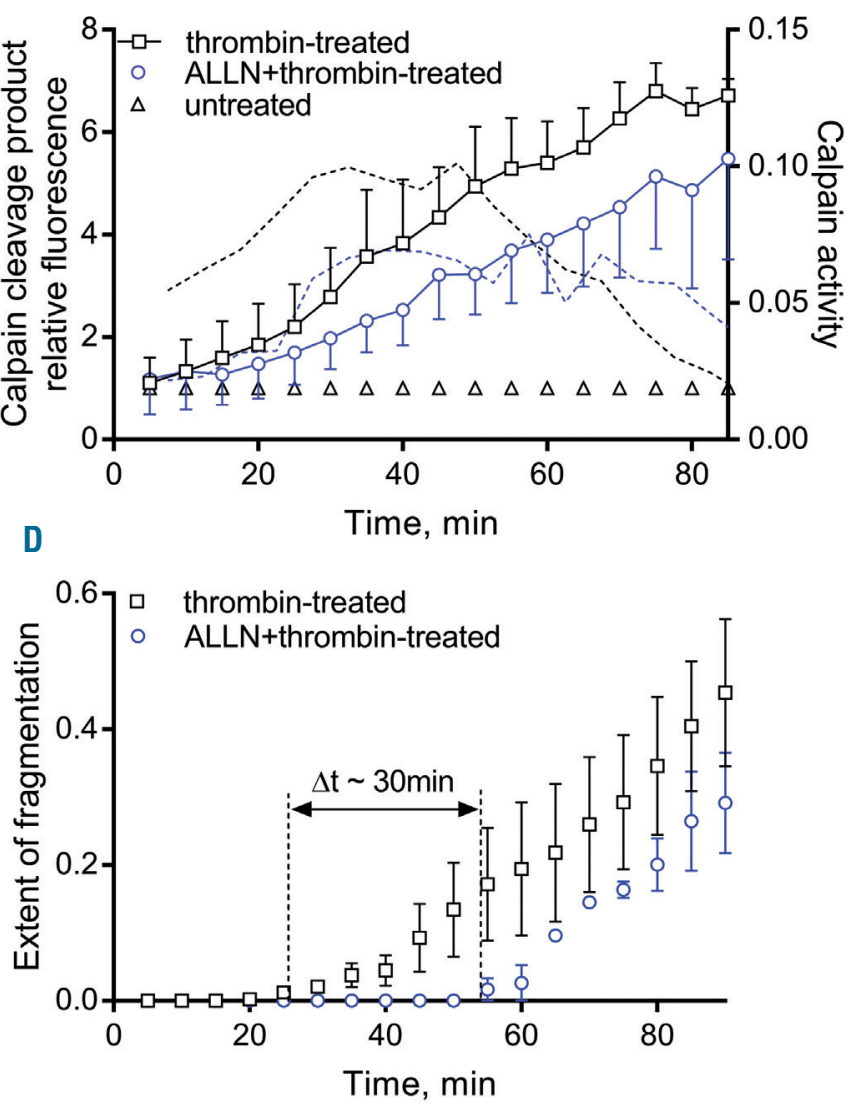

Figure 8. Calpain activation in thrombin-treated platelets and the effects of calpain inhibition on the mitochondrial membrane potential and platelet fragmentation. (A) Flow cytometry-based measurement of calpain activity assessed by the fluorogenic substrate. Isolated platelets were analyzed after 15,60 , and 180 min incubation with thrombin and the calcium ionophore $\mathrm{A} 23187$ (positive control) in the presence of $2 \mathrm{mM} \mathrm{Ca}^{2+}($ mean \pm standard deviation, $\mathrm{n}=6$ ). The data were normalized by the values for untreated platelets (negative control) at the corresponding time points (mean \pm standard error of mean, $\mathrm{n}=4$ ). $* P<0.05, * * P<0.01$, $* * * * P<0.0001$ (two-tailed Mann-Whitney U test). (B) Confocal microscopy-based time-lapse fluorescence intensity of the calpain cleavage product, reflecting calpain activity or the rate of enzymatic reaction (dashed curves, comprising the first derivative of the experimental plots). Platelets in re-calcified platelet-rich plasma (PRP) were activated with thrombin in the presence or absence of ALLN, a calpain inhibitor, and compared with untreated platelets in PRP (mean \pm standard error of mean, $\mathrm{n}=4$ ). (C) Time-lapse confocal microscopy of the MitoTracker fluorescence intensity, reflecting the platelet mitochondrial transmembrane potential in the presence or absence of ALLN during 90 min incubation with thrombin and $\mathrm{Ca}^{2+}$ at $37^{\circ} \mathrm{C}$ (mean \pm standard error of mean, $\mathrm{n}=4$ ). (D) Confocal microscopy-based platelet fragmentation dynamics in the PRP clots tracked over time in the absence or presence of ALLN (mean \pm standard error of mean, $n=4$ ). $P<0.05$ (B-D) for the difference between thrombin-treated platelets and thrombin-treated platelets in the presence of ALLN (two-tailed Mann-Whitney U test). 
stage alterations can be critical for platelet fate and may be important for remodeling and resolution of clots and thrombi. In this work, we tracked delayed structural, biochemical, and biomechanical changes in human platelets exposed to thrombin, a potent platelet stimulant in (pro)thrombotic states.

Based on the literature and our own observations, ${ }^{18,28}$ we hypothesized that after a period of thrombin-induced augmented functionality, platelets would become dysfunctional and lose their structural integrity. Our present findings support this hypothesis and shed light on the mechanisms underlying thrombin-induced platelet death. Structurally, a substantial fraction of thrombin-stimulated platelets undergo disintegration into subcellular organellecontaining fragments (Figures 1-3). This effect is specific for thrombin because no fragmentation was induced by collagen or ADP (Online Supplementary Figures S2). Thrombin-induced platelet fragmentation is associated with changes in intracellular $\mathrm{Ca}^{2+}$ concentration and reorganization of the platelet cytoskeleton (Figures 4,5). It is also concurrent with cessation of platelet contractility, metabolic ATP depletion, formation of ROS, and mitochondrial depolarization (Figure 6). Notably, thrombin does not induce detectable activation of the effector caspases 3 and 7 in platelets, suggesting a caspase-independent platelet death pathway (Figure 7).

Thrombin-treated platelets break up into extracellular particles of various sizes, origin and composition. It is tempting to identify the platelet body fragments as exosomes, but they are distinct in a number of features. First, particles formed during break up of thrombin-stimulated platelets are relatively large and heterogeneous $(0.1-1 \mu \mathrm{m})$ (Figures 1-3), whereas exosomes are smaller and more uniform $(0.03-0.10 \mu \mathrm{m})^{29}$ Second, the larger platelet fragments are produced by dysfunctional and energetically exhausted platelets, whereas exosomes are generated by metabolically active and structurally intact platelets from their endosomal multi-vesicular bodies. ${ }^{29}$ Therefore, the particles formed during thrombin-induced platelet disintegration comprise a special type of platelet-derived particles looking like "apoptotic cellular bodies" rather than membrane-derived microvesicles or secreted exosomes and are likely to be cleared by phagocytes from the blood flow. ${ }^{30}$ Furthermore, structurally, the changes observed in platelets do not resemble those that occur in necrosis since there is no swelling and no rupture of the cell membrane or that of cellular organelles and platelet fragments all retain intact membrane (Figure 3 ).

Fragmentation of thrombin-treated platelets is accompanied by a dramatic increase in F-actin-related fluorescence intensity and redistribution of F-actin towards the center of platelets with formation of highly fluorescent patches (Figure 4), suggesting polymerization and/or clustering of actin. Remarkably, the smallest platelet fragments do not show F-actin staining, suggesting that actin filaments are depolymerized or destroyed at the late stages of platelet disintegration. The essential role of F-actin dynamics in thrombin-induced platelet fragmentation is confirmed by the complete prevention of platelet disintegration after blocking actin polymerization with cytochalasin D or latrunculin A (Figure 4G,H and Online Supplementary Figure S4). The membrane cytoskeleton formed of microtubules is also involved in platelet disintegration, because inhibition of tubulin dynamics with paclitaxel prevented the break-up of platelets caused by thrombin (not shown).
Platelet disintegration induced by thrombin correlates strongly with energy exhaustion and dysfunction that includes mitochondrial depolarization, a drop in the ATP content, and the loss of contractility, all seen at about 30 min after addition of thrombin (Figure 6). The observed mitochondrial depolarization may be attributed to an increase in cytosolic and mitochondrial $\mathrm{Ca}^{2+}$ upon thrombin treatment (Figure 5), which can trigger cyclophilin Ddependent mechanisms of the mitochondrial potential collapse. ${ }^{31}$ This decrease in $\Delta \Psi_{\mathrm{m}}$ was shown to be associated with generation of ROS (Figure 6), which can damage intracellular structures and could cause platelet death. . $2,33^{-}$ Remarkably, some mitochondria in activated platelets localize in filopodia and may be released in the extracellular milieu. Although the mechanism of mitochondria translocation toward the tips of platelet filopodia is not clear, ROS-dependent actin polymerization and mitochondria-bound myosin were shown to participate in intracellular mitochondria transport. ${ }^{34,35}$ This mechanism may also be important to translocate mitochondria to sites of high ATP utilization, such as contracting filopodia.

The metabolic ATP depletion in platelets may be due to mitochondrial depolarization as well as to impaired glycolysis, both important sources of ATP in activated platelets. ${ }^{36-38}$ The insufficiency of ATP is aggravated by its consumption due to energy-demanding platelet functions, such as contractility. Irrespective of the mechanism of the decrease of ATP content, it is a signature of energy exhaustion and impaired platelet functionality. Not surprisingly, the significant $(\sim 54 \%)$ decrease of ATP content in platelets after 30 min of thrombin treatment coincided with the termination of platelet-driven clot contraction that depends on the activity of non-muscle myosin II, which is a major part of the ATP-dependent-cell contractile machinery. ${ }^{39}$ The inhibitory effect of blebbistatin on platelet fragmentation (not shown) implies that the contractile myosin IIaactin complex contributes to platelet disintegration, perhaps facilitating mechanical disconnection or dislodging of fragments. It is not clear whether the revealed biochemical and biomechanical alterations associated with platelet thrombin-induced disintegration provide the conditions for the structural decomposition of platelets or comprise its consequences. However, it is evident that the magnitude and kinetics of the observed late-stage functional and metabolic alterations following thrombin treatment of platelets are distinct from the non-specific changes related to platelet aging or "storage lesions" (see the Online Supplementary Results section).

Neither time-lapse confocal microscopy, nor flow cytometry nor western blotting detected procaspase 3/7 activation in platelets in response to thrombin stimulation (Figure 7), indicating that the thrombin-induced platelet death pathway is caspase-independent and is likely nonapoptotic, which is in agreement with previous results ${ }^{40}$ that demonstrated no caspase- 3 activity in platelets treated with thrombin. Alternatively, our data suggest the involvement of $\mathrm{Ca}^{2+}$-dependent protease calpain in platelet disintegration, perhaps due to calpain-catalyzed cleavage of cytoskeletal proteins. ${ }^{41}$ Calpain may compensate for the lack of active caspases by cleaving caspase substrates such as gelsolin, protein kinase C- $\delta$ and fodrin. It can also cleave other cytosolic proteins and many important regulators of apoptosis, including the anti-apoptotic XIAP and $\mathrm{Bcl}-\mathrm{xL}$, thereby recapitulating apoptotic events in activated platelets. ${ }^{26,42-44}$ For most cells, changes in the nucleus are 
an essential part of apoptosis, but for the anucleate platelet the cell's development and lifespan are determined by the intrinsic pathway of apoptosis. ${ }^{19}$ On the other hand, whether or not apoptosis is involved in the fate of platelets after activation has been controversial, but other death pathways cannot be excluded..$^{45}$

Irrespective of the mechanisms underlying platelet death as an outcome of thrombin-induced activation, our data suggest a pathway for enhanced elimination of activated platelets from the circulation in (pro)thrombotic conditions associated with thrombinemia. In severe thrombotic conditions, such as disseminated intravascular coagulation $^{46}$ or trauma-induced coagulopathy, ${ }^{47}$ platelets may vanish due to removal of platelet fragments from the blood, perhaps by monocytes, dendritic cells and macrophages. ${ }^{48}$ Thrombin-induced platelet disintegration may therefore be a pathogenic mechanism that modulates platelet counts, functionality, and fate in disease states associated with hypercoagulability and high thrombin activity in blood.

In conclusion, following thrombin-induced platelet activation, a substantial fraction of platelets later undergo structural disintegration into subcellular particles. This fragmentation of platelets is accompanied by dramatic rearrangements of platelet cytoskeletal components, including redistribution of actin and microtubule dynamics. Thrombin-induced platelet fragmentation is concur- rent with severe impairment of platelet functionality, including mitochondrial depolarization, metabolic ATP depletion, generation of ROS, and loss of platelet contractility. The lack of caspase activity and increased calpain activity in energy-exhausted thrombin-treated platelets undergoing fragmentation suggests a calpain-dependent platelet death pathway. Fragmentation of activated platelets suggests that platelet death is an underappreciated mechanism for enhanced elimination of platelets from the circulation in (pro)thrombotic conditions or under other conditions once these cells have performed their functions. Analogous to eryptosis, suicidal death of erythrocytes, the platelet death pathway described here could be named "thromboptosis" or "plateleptosis".

\section{Acknowledgments}

We would like to thank Dr. Xiaolu Yang for fruitful discussions. The work was supported by National Institutes of Health grants UO1HL116330 and R01 HL135254, National Science Foundation grant DMR1505662, the Program for Competitive Growth at Kazan Federal University, grant 18-415-160004 from the Russian Foundation for Basic Research, American Heart Association grants $17 S D G 33680177$ and 16PRE30260002, and grant EPSRC EP/C513037/1 to P. R. Williams (Swansea University, Wales, UK) for the TA Instruments ARG2 rheometer. Transmission electron microscopy was carried out on the equipment of CSF-SAC FRC KSC RAS (Kazan, Russia).

\section{References}

1. Rosen ED, Raymond S, Zollman A, et al. Laser-induced noninvasive vascular injury models in mice generate platelet-and coagulation-dependent thrombi. Am J Pathol. 2001;158(5):1613-1622.

2. Brass LF, Ma P, Tomaiuolo M, Diamond SL, Stalker TJ. A systems approach to the platelet signaling network and the hemostatic response to injury. In: Platelets in Thrombotic and Non-Thrombotic Disorders. Springer. 2017; p.367-378.

3. Kim OV, Litvinov RI, Alber MS, Weisel JW. Quantitative structural mechanobiology of platelet-driven blood clot contraction. Nat Commun. 2017;8(1):1274.

4. Le Minh G, Peshkova AD, Andrianova IA, et al. Impaired contraction of blood clots as a novel prothrombotic mechanism in systemic lupus erythematosus. Clin Sci. 2018;132(2):243-254.

5. Peshkova AD, Minh GL, Tutwiler V, Andrianova IA, Weisel JW, Litvinov RI. Activated monocytes enhance platelet-driven contraction of blood clots via tissuefactor expression. Sci Rep. 2017;7(1):5149.

6. Tutwiler V, Wang H, Litvinov RI, Weisel JW, Shenoy VB. Interplay of platelet contractility and elasticity of fibrin/erythrocytes in blood clot retraction. Biophys J. 2017;112(4):714723.

7. Kamath S, Blann A, Lip G. Platelet activation: assessment and quantification. Eur Heart J. 2001;22(17):1561-1571.

8. Prevost N, Kato H, Bodin L, Shattil SJ. Platelet integrin adhesive functions and signaling. Methods Enzymol. 2007;426:103-115.

9. Shattil SJ. Integrins and Src: dynamic duo of adhesion signaling. Trends Cell Biol. 2005;15(8):399-403.
10. Siess W. Molecular mechanisms of platelet activation. Physiol Rev. 1989;69(1):58-178.

11. Pang A, Cui Y, Chen Y, et al. Shear-induced integrin signaling in platelet phosphatidylserine exposure, microvesicle release and coagulation. Blood. 2018;132 (5):533-543

12. Aslan JE. Platelet shape change. In: Platelets in Thrombotic and Non-Thrombotic Disorders. Springer. 2017;p 321-336.

13. Shin E-K, Park H, Noh J-Y, Lim K-M, Chung J-H. Platelet shape changes and cytoskeleton dynamics as novel therapeutic targets for anti-thrombotic drugs. Biomol Ther. 2017;25(3):223-230.

14. Seifert J, Rheinlaender J, Lang F, Gawaz M Schäffer TE. Thrombin-induced cytoskeleton dynamics in spread human platelets observed with fast scanning ion conduc tance microscopy. Sci Rep. 2017;7(1):4810.

15. Sandmann R, Köster S. Topographic cues reveal two distinct spreading mechanisms in blood platelets. Sci Rep. 2016;6:22357.

16. Diagouraga B, Grichine A, Fertin A, Wang J, Khochbin S, Sadoul K. Motor-driven marginal band coiling promotes cell shape change during platelet activation. J Cell Biol. 2014;204(2):177-185

17. Edelstein LC. The role of platelet microvesicles in intercellular communication. Platelets. 2017;28(3):222-227.

18. Ponomareva A, Nevzorova T, Mordakhanova E, et al. Intracellular origin and ultrastructure of platelet-derived microparticles. J Thromb Haemost. 2017:15(8):1655-1667.

19. Mason KD, Carpinelli MR, Fletcher JI, et al Programmed anuclear cell death delimits platelet life span. Cell. 2007;128(6):11731186.

20. Oltersdorf T, Elmore SW, Shoemaker AR, et al. An inhibitor of Bcl-2 family proteins induces regression of solid tumours. Nature. 2005;435(7042):677-681.

21. McArthur K, Chappaz S, Kile BT. Apoptosis in megakaryocytes and platelets: life and death of a lineage. Blood. 2018;131(12):605610.

22. Beigi R, Kobatake E, Aizawa M, Dubyak GR. Detection of local ATP release from activated platelets using cell surface-attached firefly luciferase. Am J Physiol. 1999;276(1):C267-C278.

23. Leytin V, Allen DJ, Mykhaylov S, Lyubimov E, Freedman J. Thrombin-triggered platelet apoptosis. J Thromb Haemost. 2006;4(12):2656-2663.

24. Leytin V. Apoptosis in the anucleate platelet. Blood Rev. 2012;26(2):51-63.

25. White MJ, Kile BT. Apoptotic processes in megakaryocytes and platelets. In: Seminars in Hematology. Elsevier. 2010;p 227-234.

26. Vanags DM, Pörn-Ares MI, Coppola S, Burgess DH, Orrenius S. Protease involvement in fodrin cleavage and phosphatidylserine exposure in apoptosis. J Biol Chem. 1996;271(49):31075-31085.

27. Wood D, Thomas A A, Devi L, et al. Bax cleavage is mediated by calpain during drug induced apoptosis. Oncogene. 1998;17(9): 1069-1078.

28. Agbani EO, Williams CM, Hers I, Poole AW Membrane ballooning in aggregated platelets is synchronised and mediates a surge in microvesiculation. Sci Rep. 2017;7(1):2770

29. Heijnen HF, Schiel AE, Fijnheer R, Geuze HJ Sixma JJ. Activated platelets release two types of membrane vesicles: microvesicles by surface shedding and exosomes derived from exocytosis of multivesicular bodies and alfa-granules. Blood. 1999;94(11):37913799

30. Brown SB, Clarke MCH, Magowan L, 
Sanderson H, Savill J. Constitutive death of platelets leading to scavenger receptor-mediated phagocytosis. A caspase-independent cell clearance program. J Biol Chem. 2000;275(8):5987-5996.

31. Jobe SM, Wilson KM, Leo L, et al. Critical role for the mitochondrial permeability transition pore and cyclophilin $\mathrm{D}$ in platelet activation and thrombosis. Blood. 2008;111(3): 1257-1265.

32. Choo H-J, Saafir TB, Mkumba L, Wagner MB, Jobe SM. Mitochondrial calcium and reactive oxygen species regulate agonist-initiated platelet phosphatidylserine exposure. Arterioscler Thromb Vasc Biol. 2012;32(12):2946-2955.

33. Yamagishi S, Edelstein D, Du X, Brownlee M. Hyperglycemia potentiates collageninduced platelet activation through mitochondrial superoxide overproduction. Diabetes. 2001;50(6):1491-1494.

34. Shneyer BI, Ušaj M, Wiesel-Motiuk N, Regev R, Henn A. ROS induced distribution of mitochondria to filopodia by Myo19 depends on a class specific tryptophan in the motor domain. Sci Rep. 2017;7(1):11577.

35. Boudreau LH, Duchez A-C, Cloutier N, et al. Platelets release mitochondria serving as substrate for bactericidal group IIA-secreted phospholipase A2 to promote inflammation. Blood. 2014;124(14):2173-2183.

36. Aibibula M, Naseem KM, Sturmey RG. Glucose metabolism and metabolic flexibility in blood platelets. J Thromb Haemost. 2018;16(11):2300-2314.

37. Ravi S, Chacko B, Sawada H, et al Metabolic plasticity in resting and thrombin activated platelets. PLoS One. 2015;10(4): e0123597.

38. Nayak MK, Dhanesha N, Doddapattar P, et al. Dichloroacetate, an inhibitor of pyruvate dehydrogenase kinases, inhibits platelet aggregation and arterial thrombosis. Blood Adv. 2018;2(15):2029-2038.

39. Adelstein RS, Eisenberg E. Regulation and kinetics of the actin-myosin-ATP interaction. Annu Rev Biochem. 1980;49(1):921956.

40. Wolf BB, Goldstein JC, Stennicke HR, et al. Calpain functions in a caspase-independent manner to promote apoptosis-like events during platelet activation. Blood. 1999;94(5): 1683-1692.

41. Goll DE, Thompson VF, Li H, Wei W, Cong J. The calpain system. Physiol Rev. 2003;83(3):731-801.
42. Martin SJ, O'Brien GA, Nishioka WK, et al Proteolysis of fodrin (non-erythroid spectrin) during apoptosis. J Biol Chem 1995;270(12):6425-6428.

43. Kothakota S, Azuma T, Reinhard C, et al Caspase-3-generated fragment of gelsolin: effector of morphological change in apoptosis. Science. 1997;278(5336):294-298.

44. Harwood SM, Yaqoob MM, Allen DA Caspase and calpain function in cell death bridging the gap between apoptosis and necrosis. Ann Clin Biochem. 2005;42(6) 415-431.

45. Nogusa S, Thapa RJ, Dillon CP, et al. RIPK3 activates parallel pathways of MLKL-driven necroptosis and FADD-mediated apoptosis to protect against influenza A virus. Cell Host Microbe. 2016;20(1):13-24.

46. Gando S, Levi M, Toh C-H. Disseminated intravascular coagulation. Nat Rev Dis Primer. 2016;2:16037.

47. Chang R, Cardenas JC, Wade CE, Holcomb JB. Advances in the understanding of trauma-induced coagulopathy. Blood. 2016;128 (8):1043-1049.

48. Elliott MR, Ravichandran KS. Clearance of apoptotic cells: implications in health and disease. J Cell Biol. 2010;189(7):1059-1070. 